\title{
Maize Grain Quality Classification Using Convolutional Neural Network
}

\author{
Ashok kumar P M ${ }^{\mathrm{a}, 1}$, Anitha $\mathrm{R}^{\mathrm{a}}$ and Revathi $\mathrm{B}^{\mathrm{a}}$ \\ ${ }^{a}$ Department of Computer Science and Engineering, $K$ L Deemed to be University, \\ Green Fields, Vaddeswaram, Guntur District, A.P., India
}

\begin{abstract}
The major goal of our research would be to classify the quality of maize grain after obtaining the crop output. Grains have been the most important grain for farmers to boost their farming revenue in a country. One of the most significant food crops is wheat grain. The majority of the country's grain is used for animal feed, human consumption, and the creation of industrial products. The amount of mechanization used to assess grain quality is modest, and the majority of the job is done by hand. Every day, different prices are introduced in various marketplaces, and farmers face losses every year quality of service estimate and market rates. With our classification we can give accurate progress on the seed which will help farmer's to sell with better price along with that this assumption can idealize him to give good nutritious fertilizer to the crop.
\end{abstract}

Keywords. Convolutional Neural Networks (CNN), Deep Learning, VGG-16, VGG-19 Architectures.

\section{Introduction}

Agriculture will be incredibly efficient if it is combined with high-quality produce and competitive platforms where the produce is valued at the highest level. Grains [1] are the most important crop for farmers to grow in order to boost their agricultural revenue. One of the really important cereal crops is maize grain [2]. It is one of the most adaptable growing harvests, allowing it to thrive in a variety of agricultural production situations. The mechanization level of maize crop assessment [3] is tough, and the majority of the job is done by hand. The majority of maize is utilised for livestock feed, public consumption, and a variety of industrial goods. Mechanical goods have advanced rapidly in recent decades [4].

The method of wheat crop guide association is modest, has limited repeatability, and has a degree of sensitivity that is difficult to assess, both in terms of commercial enterprise and technological consequences. Visual inspection of grain seed is tedious and time-eating process, this can motive problems which include eyesight, intellectual state, paintings stress etc. In diverse markets distinctive fees [5] are introducing for each day, with the exceptional assumption and with the marketplace fees farmers are going through losses each year. These responsibilities require automation and create imaging frame-

\footnotetext{
${ }^{1}$ Ashok kumar PM, Department of CSE, K L Deemed to be University, Green Fields, Vaddeswaram.

E-mail: profpmashok@gmail.com.
} 
works that may be beneficial to differentiate maize grain. These troubles may be decreased with the adoption of latest superior technologies withinside the subject of Computer Science which makes our lives less complicated and solves complicated problems. These upgrades boom the performance and decreases human intervention. The field of image analysis in machine learning has also expanded and demonstrated its benefits in resolving a wide range of issues related to agriculture.

Transfer learning [6] is one method which makes use of the preceding information of a few different projects and attempts to resolve a awesome project with an alternate in primary intuition. This painting illustrates and compares diverse neural net-paintings architectures which offers the first-class outcomes in classifying maize corn classes.

\section{Related Works}

Cereals are one of the most important agricultural products that go into making a wide range of cuisines. The evaluation of wheat yield is determined by a variety of factors, including aspect, texture, brightness, aroma, taste, water temperature, infection, pollutant presence, and so on. Several types of grains are considered impurities: crushed grains, which account for less than half of the total grains; crushed grains, account for half of the total grains. Small, wrinkled, and green grains; sprouted grains, grains with moldy germs, ranging from dark deep violet; Fusarium infects wheat. Non-grain contaminants include: damaged grains, corn grains on the cob, parts of leaves and stems, pebbles, dirt, sand, obscene grains, and harmful elements.

Numerous comes about have been distributed, in which color characteristics examination was utilized to survey a few specific quality highlights. To determine the hue or tone group mixture that offered the most notable categorization correctness in evaluating the real-ness of wheat flour, the textural properties of individual bits were extracted from various colors and color band combinations of images. hue descriptors interpretation was utilized too to survey course [7], diseases [8], germi-nation [9], weed distinguishing proof [10], etc.Also the system designed by [11] offered higher accuracy based on optimiztion in real time. It used Fast R-CNN which adapts to complex and rapid flying environment changes.

Evaluation of the grain test immaculateness was performed by profile examination of corn parts utilizing 1-D computerized signals based on its double pictures [12], by simulating the geometry using a set of physiological highlights [13] and by shape curvature examination [14]. Computer vision strategies were too utilized for assurance of distinctive wounds: corn part mechanical harm and form harm [15], entire and broken bit identification [16], etc.

Diverse approaches were utilized for this reason. A few successful comes about were gotten utilizing classification based on multi-layer neural systems (NN) [17]. In [18], the recognition rate of 9 artificial neural architectures was tested to categorize 5 different types of wheat flour. When interpreting a comparative assignment, [19] showed a difference between a four-layer backpropagation neural network and a pseudo factual predictor. The results show that the neural net predictor outperformed the non-parametric classifier in almost every categorization situation.

The fineness of wheat test components was analyzed using neural systems in conjunction with hue, contour, and size of the items. They are often used to examine the 
lesson, virtue, various mechanical damages, germination, contaminations, and weed seed detection, among other things.

\section{Analysis of Maize Grain}

\subsection{Theoretical Analysis}

Image popularity is the method of classifying the photograph primarily based totally at the data. Image popularity is used to carry out a massive range of machine-primarily based totally tasks, like content material of photos labelling with meta- tags, appearing photograph search, guiding the cus-tomers, even in a few instances self-riding vehicle and coincidence fending off structures etc., whilst human brains apprehend gadgets with ease, computer systems have issue with the task. Software for photograph popularity calls for deep mastering techniques.

Performance is likewise excellent with the use of of convolution neural network. For our device maize grading, we're the use of convolution neural networks for grading maize grains. Wheat seems to be the most agricultural commodity for villagers to increase overall agricultural revenue. Wheat grain is among the most abundant cereal crops. This are among the most adaptable developing yields, allowing it to thrive in a variety of different agricultural situations.

The robotic stage rating $[9,10]$ of maize grains is difficult, and the majority of the work is done manually. The majority of the maize harvest is utilized for livestock feed, public consumption, and a variety of commercial items. Machine tools have advanced fast in recent years. The process of guide association of wheat flour is mild, has low repeatability, and has a stage of subjectivity difficult to assess, each in its commercial enterprise in addition to scientific ramifications.

Microscopic inspection of wheat crop [12,13] is a tedious and time-eating process, this will reason troubles which include eyesight, intellectual state, paintings strain etc. In diverse markets unique costs are introducing for each day, with the fine assumption and with the marketplace costs farmers are dealing with losses each year. These duties require au-tomation $[14,15]$ and create 17 imaging frame-works that may be beneficial to differentiate maize grain. These troubles may be decreased with the adoption of latest superior technology withinside the discipline of Computer Science which makes our lives less complicated and solves complicated troubles.

\subsection{Statistical Analysis}

Data evaluation and facts are extraordinarily im-portant to many fields. In fact, facts and statistics evaluation are important for undertaking inner audits and overall performance reviews. In many industries like marketing, research, monetary offerings and medical or medical research, facts and statistics 6 analysis are important. This is authentic due to the inherent strength of facts withinside the reading of statistics.

Statistics also can be vital to attain extremely vital boom and performance objectives. Because it's so vital for corporations to apply information to result in significant outcomes, interpretations and enterprise decisions, the significance of information can't be overstated. Statistical Analysis assist can play a pivotal position in any task regarding 
information as Statistical Analysis Help produces excessive exceptional outcomes irrespective of the sphere or concern matter. We gathered this data from Agricultural Department. The count value in various markets are different due to various reasons like types of crop, irrigation, transportation and the irrigation.

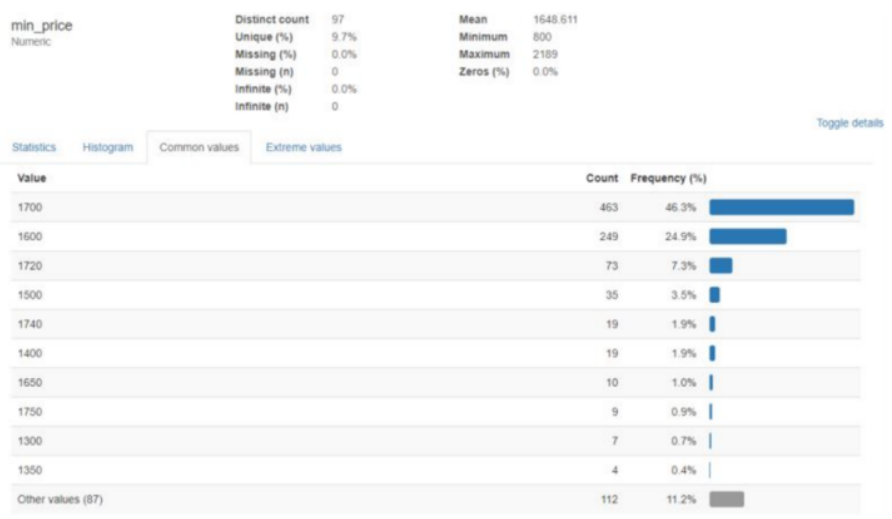

Figure 1. Word Frequency Count

From the above table it clarifies that where the count value is high the frequency of the crop is also high. In these markets overall all crops in India is traded but maize is traded more. There are 8 markets in which 2 markets are not good with maize crop, the count values of other markets are raises in between $103-210$. This area has high yield growth, with this marketing frequency is also high. Various types of crops are marketing in different markets.

Unique crop marketing is compared less which approximately 0.8 Above data is representing the prices all over the nation. The prices in various markets are similar/different. The estimation of price is from $800-1700$ based on the yield of the crop and the availability of crop in that area. If the demand is high and the stock is less then automatically prices go up. The overall mean average price of the crop in our country is 1648.611 and the maximum it reaches is 2189 .

\section{Methodology}

\subsection{Data Description}

The collection contains wheat grain images gathered from the Andhra Pradesh Agriculture Department in India. Throughout the data collection, wheat grain pictures were categorised into five categories depending on their excellence. Excellent, Good, Average, $\mathrm{Bad}$, and Worst are among the directions. The dataset includes 2500 pictures wherein every class includes 500 pictures resembles a balanced dataset. The below image depicts the different categories of the maize corn grains. 


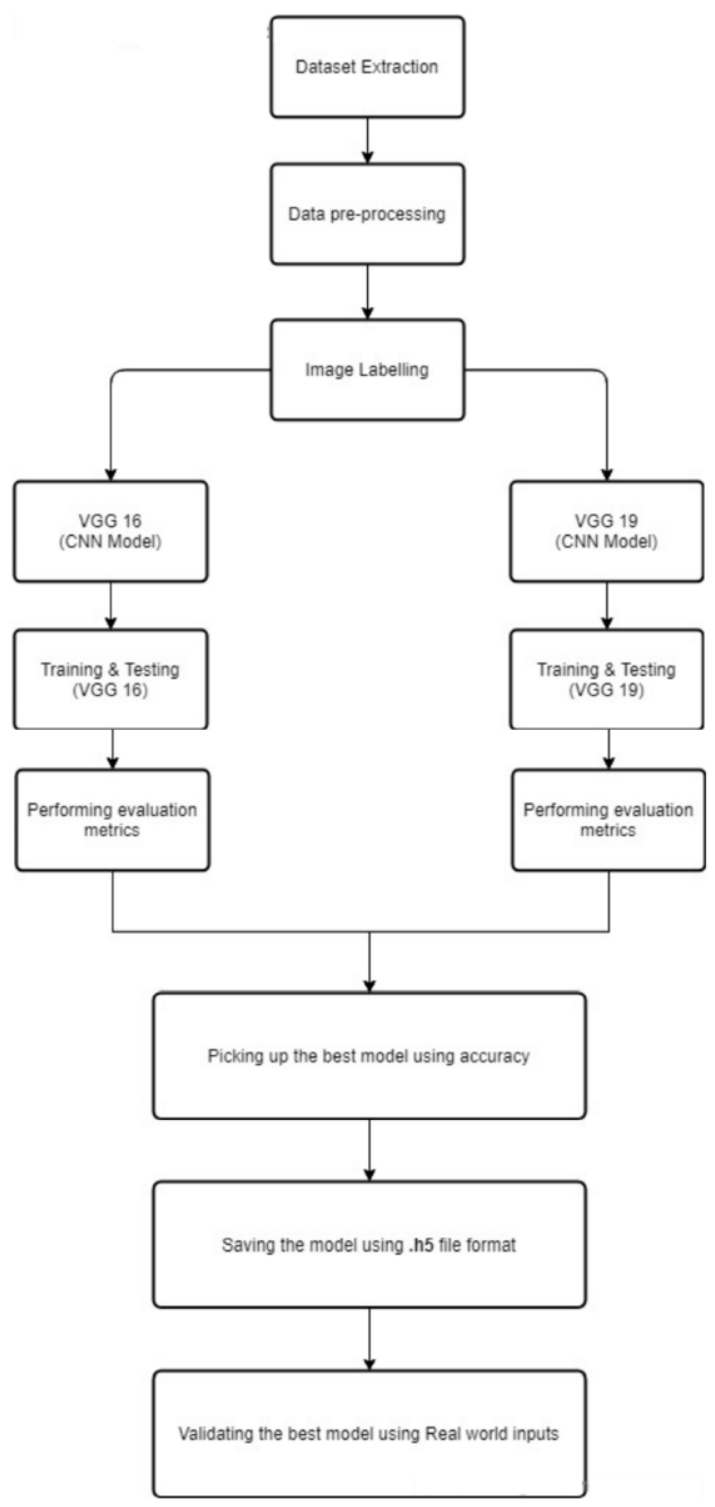

Figure 2. Proposed Methodology

\subsection{Data Pre-Processing}

In actual global it's far very tough to put together exceptional photo facts for education a neural network. The preliminary stage requires a lot of hard to make the difference in producing improved result. The pics in our dataset are of very large proportions. As a result, all of the photos have been downsized to 256x256 pixels. The 10 photos are down sampled and adjusted, making them easier for the neural net to understand. Gen- 
erally, Deep Learning Models calls for large quantity of facts to per-shape well. Since the dataset includes much less quantity of facts, we implemented photo augmentation approach. As a consequence of this approach's software, photos are synthetized using various techniques, demonstrated that using this approach also prevents the version from over becoming, which in turn adjusts the error value at each cycle. The image data is

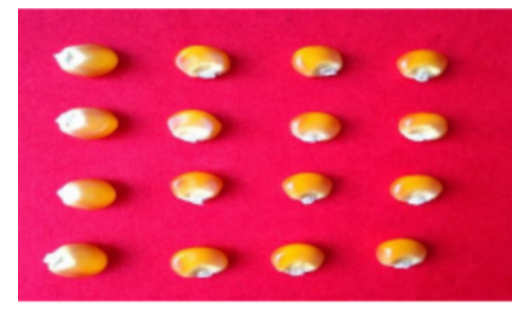

(a) Excellent

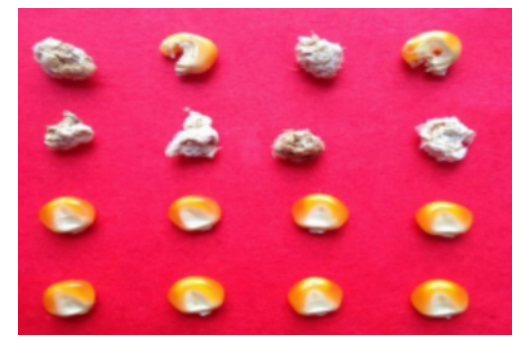

(c) Average

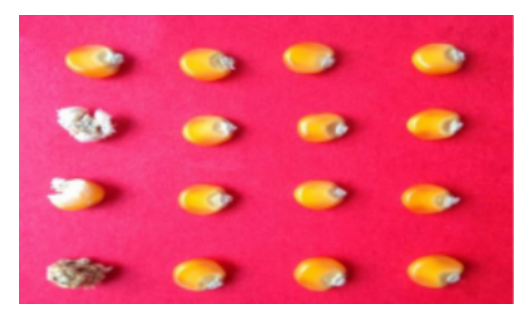

(b) Good

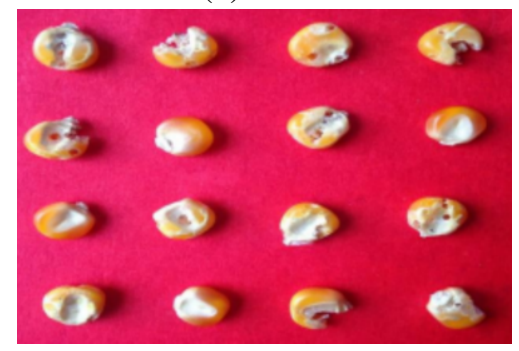

(d) Bad

Figure 3. Different types of Maize Grain Quality

sent to multiple neural network designs, including VGG-16, VGG-19, InceptionV3, and Resnet50, after the preliminary stage. The utility of switch gaining knowledge of strategies in those neu ral networks architectures makes using weights of every layer which might be skilled on Image internet Dataset includes one thousand classes. We removed the bottom layer weights and re-trained on other architectures because the instinct of the problem is distinct. Categorical move entropy is the loss function used in neural network community, while Stochastic Gradient Descent is the optimizer (SGD). We ran the data collection through various designs using the measure of specified detection rate as the measure.

\subsection{Convolutional Neural Network (CNN)}

Convolutional Neural Networks are very just like regular Neural Networks they're created from neurons which have learnable hundreds and inclinations. Each neuron gets a few inputs, plays a dot product and optionally follows it with a non-linearity The complete community nonetheless expresses a single differentiable rating function: from the uncooked photograph pixels on one quit to elegance ratings on the other. And they nonetheless have a loss function (e.g. SVM/SoftMax) at the last (absolutely connected) layer and all of the tips/hints we advanced for getting to know every day Neural Networks nonetheless apply. .ConvNet architectures make the specific assumption that the inputs are images, which lets in us to encode positive houses into the architecture. These 


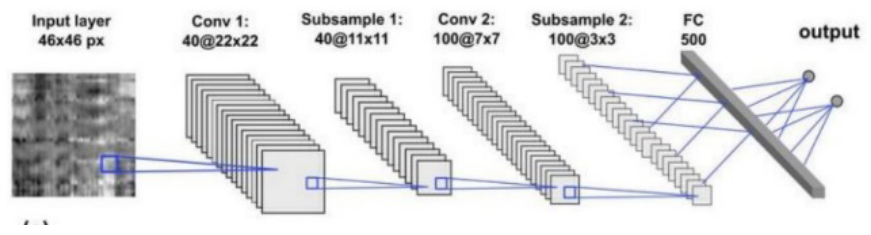

Figure 4. Convolution Network Architecture

then make the ahead characteristic extra efficient to put into effect and massively lessen the wide variety of parameters withinside the network.
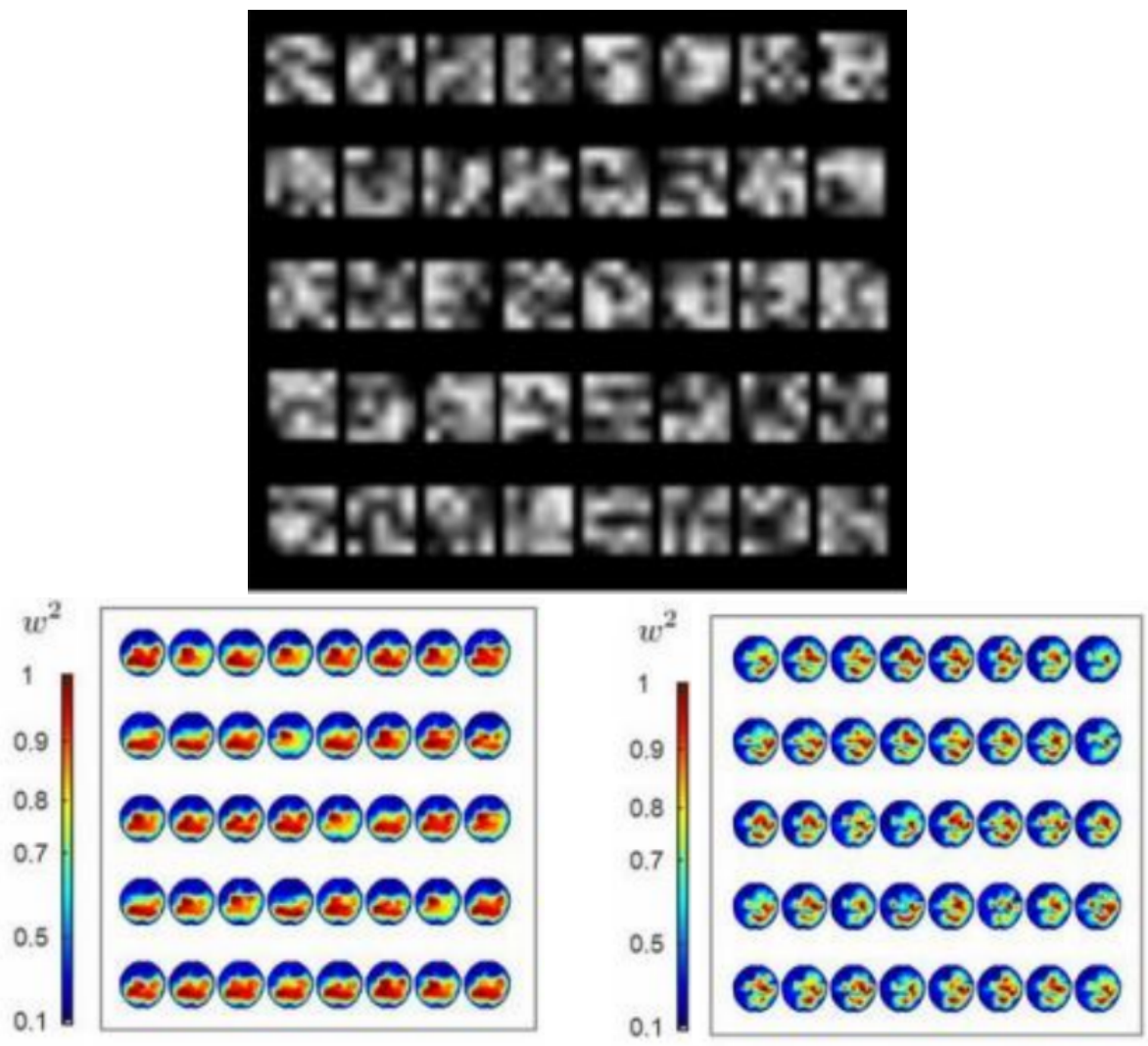

Figure 5. Implementation Result

\subsubsection{VGG-16 CNN Model}

VGG16 is a convolutional neural net (CNN) architecture that was utilized to win the 2014 ILSVR(ImageNet) competition. It is regarded amongst the most inventive and foresightful version structures ever created. The most distinctive feature of VGG16 is that, rather than obtaining a large number of hyper-parameters, they focused on creating 26 levels of $3 \times 3$ clear out with a step 1 and al-approaches employed similar padding as well as a max-pool level of $2 \times 2$ clear out of step 2 . For the lifespan of the structure, it 
maintains this relationship between convolution and max pool levels. In the cease it has 2 FC (completely linked layers) observed through a soft-max for results. The sixteen in VGG16 alludes to the fact that it has 16- weighted levels. This community is a pretty massive community and it has approximately 138 million (approx.) parameters.

\subsubsection{VGG-19 CNN Model}

In this research, we look at the impact of the convolutional community intensity on its accuracy withinside the large-scale photo popularity setting. Our most important contribution is a radical assessment of networks of growing intensity the use of an structure with very small $(3 \times 3)$ convolution filters, which suggests that a vast development at the prior-artwork configu-rations may be executed with the aid of using pushing the intensity to 16-19 weight layers. These findings have been the idea of our ImageNet Challenge 2014 submission, wherein our crew secured the primary and the second one locations withinside the localization and category tracks respectively. We additionally display that our representations generalize nicely to different datasets, wherein they reap state-of-the artwork results. We have made our best-acting ConvNet fashions publicly to be had to facilitate similarly studies on using deep visible representations in pc vision.

\section{Results and Analysis}

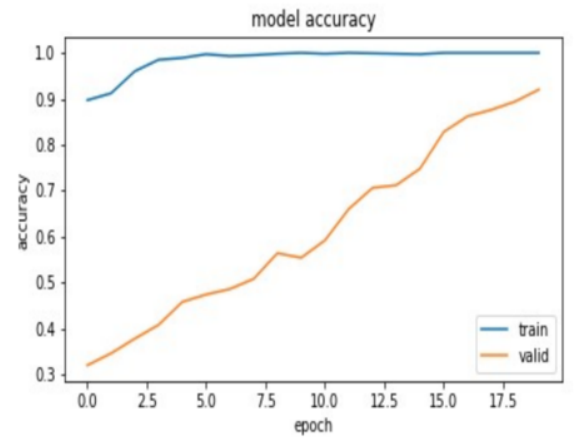

Figure 6. Model Accuracy of VGG-16

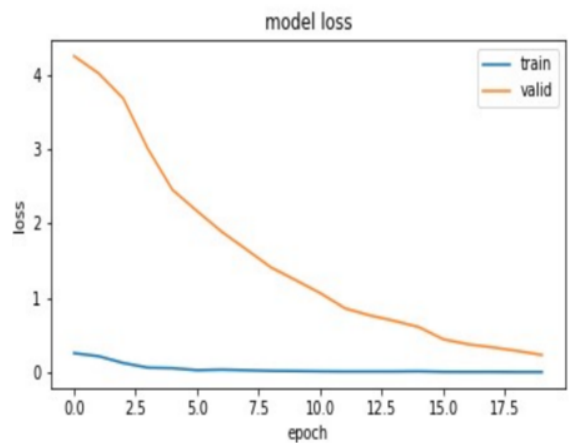

Figure 7. Model Loss of VGG-16

The above desk suggests the explicit accuracies of VGG16 VGG-19 architectures. It may be inferred from the desk that VGG-sixteen structure offers the fine overall performance in comparison to VGG-19 structure in grading the maize classification. So, the adoption of VGG-sixteen structure with the assist of switch gaining knowledge of method offers the fine results.

Table 1. Average Accuracy and Loss Values of VGG-16 and VGG-19

\begin{tabular}{|l|c|c|}
\hline Model & Accuracy (\%) & Loss (\%) \\
\hline VGG-16 & 92 & 08 \\
\hline VGG-19 & 90 & 10 \\
\hline
\end{tabular}




\section{References}

[1] OuYang AG, Gao RJ, Sun XD, Pan YY, Dong XL. An automatic method for identifying different variety of rice seeds using machine vision technology. In 2010 Sixth International Conference on Natural Computation 2010 Aug 10 (Vol. 1, pp. 84-88). IEEE.

[2] Parmar RR, Jain KR, Modi CK. Image morphological operation based quality analysis of coriander seed (Coriandrum satavum L). In 2011 International Conference on Emerging Trends in Networks and Computer Communications (ETNCC) 2011 Apr 22 (pp. 482-486). IEEE.

[3] Xun Y, Zhang J, Li W, Cai W. Automatic system of seeds refined grading based on machine vision. In 2006 6th World Congress on Intelligent Control and Automation 2006 Jun 21 (Vol. 2, pp. 9686-9689). IEEE.

[4] Yuanyuan Z, Jilong Z, Lingshen F. Research on quality evaluation of maize seed shape based on support vector machine. In 2016 2nd IEEE International Conference on Computer and Communications (ICCC) 2016 Oct 14 (pp. 695-699). IEEE.

[5] Yi X, Eramian M, Wang R, Neufeld E. Identification of morphologically similar seeds using multi-kernel learning. In 2014 Canadian Conference on Computer and Robot Vision 2014 May 6 (pp. 143-150). IEEE.

[6] Khunkhett S, Remsungnen T. Non-destructive identification of pure breeding rice seed using digital image analysis. In 4th Joint International Conference on Information and Communication Technology, Electronic and Electrical Engineering (JICTEE) 2014 Mar 5 (pp. 1-4). IEEE.

[7] Khoenkaw P. An image-processing based algorithm for rice seed germination rate evaluation. In 2016 International Computer Science and Engineering Conference (ICSEC) 2016 Dec 14 (pp. 1-5). IEEE.

[8] Hashim H, Osman FN, Al Junid SA, Haron MA, Salleh HM. An intelligent classification model for rubber seed clones based on shape features through imaging techniques. In 2010 International Conference on Intelligent Systems, Modelling and Simulation 2010 Jan 27 (pp. 25-31). IEEE.

[9] Han SH, Lee KY. Implemetation of image classification CNN using multi thread GPU. In 2017 International SoC Design Conference (ISOCC) 2017 Nov 5 (pp. 296-297). IEEE.

[10] Paliwal J, Visen NS, Jayas DS. Evaluation of neural network architectures for cereal grain classification using morphological features. Journal of agricultural engineering research. 2001;79(4):361-70.

[11] Yang R. UAV landmark detection on fast region-based CNN. Arabian Journal of Geosciences. 2021 Jun;14(12):1-9.

[12] Paliwal J, Visen NS, Jayas DS, White ND. Cereal grain and dockage identification using machine vision. Biosystems engineering. 2003 May 1;85(1):51-7.

[13] Aitkenhead MJ, Dalgetty IA, Mullins CE, McDonald AJ, Strachan NJ. Weed and crop discrimination using image analysis and artificial intelligence methods. Computers and electronics in Agriculture. 2003 Aug 1;39(3):157-71.

[14] Mladenov M, Dejanov M. Application of neural networks for seed germination assessment. In Proceedings of the 9th WSEAS international conference on Neural Networks CNN 2008 May 2 (Vol. 8, pp. 67-72).

[15] Mladenov M, Penchev S, Dejanov M, Mustafa M. Quality assessment of grain samples using color image analysis. choice. $2011 \mathrm{Feb}$ 16;1(3):37-44.

[16] Majumdar S, Jayas DS. Classification of cereal grains using machine vision: III. Texture models. Transactions of the ASAE. 2000;43(6):1681-7.

[17] Sabena S, Kalaiselvi S, Anusha B, Ramesh LS. An Multi-Label Classification with Label Correlation. Asian Journal of Research in Social Sciences and Humanities. 2016;6(9):373-86.

[18] Sabena S, Sai Ramesh L, Selva Kumar K. Multiple Cancer's Prediction using Hybrid Naïve Baye's Classifier. Asian Journal of Research in Social Sciences and Humanities. 2016;6(6):1770-82.

[19] Mittal A, Soorya A, Nagrath P, Hemanth DJ. Data augmentation based morphological classification of galaxies using deep convolutional neural network. Earth Science Informatics. 2019 Dec 9;13:1-7. 